\title{
Value of diffusion-weighted imaging in preoperative evaluation and prediction of postoperative supplementary therapy for patients with cervical cancer
}

\author{
Liying Liu ${ }^{1 \#}$, Shuo Wang ${ }^{1 \#}$, Tao Yu ${ }^{2}$, Haoyan Bai ${ }^{2}$ Jingyu Liu ${ }^{2}$, Danbo Wang ${ }^{1,3}$, Yahong Luo ${ }^{2}$ \\ ${ }^{1}$ Department of Gynecology, Cancer Hospital of China Medical University, Shenyang, China; ${ }^{2}$ Department of Radiology and Nuclear Medicine, \\ Cancer Hospital of China Medical University, Shenyang, China; ${ }^{3}$ Liaoning Cancer Institute, Shenyang, China \\ Contributions: (I) Conception and design: L Liu, D Wang; (II) Administrative support: D Wang, Y Luo; (III) Provision of study materials or patients: \\ T Yu, J Liu, H Bai; (IV) Collection and assembly of data: L Liu, S Wang; (V) Data analysis and interpretation: L Liu, D Wang; (VI) Manuscript \\ writing: All authors; (VII) Final approval of manuscript: All authors. \\ \#These authors contributed equally to this work. \\ Correspondence to: Danbo Wang; Yahong Luo. Cancer Hospital of China Medical University, 44 Xiaoheyan Rd., Dadong District, Shenyang 110042, \\ China. Email: wangdanbo@cancerhosp-ln-cmu.com; luoyahong8888@hotmail.com.
}

Background: With the continuous progress of medical imaging technology, evaluation of cervical cancer is increasingly dependent on imaging methods. Diffusion-weighted imaging (DWI) plays an important role, and apparent diffusion coefficient (ADC) value is a unique quantitative parameter in the research of cervical cancer.

Methods: In this prospective study, a total of 273 patients diagnosed with stage IB1 to IIIC1 cervical cancer based on the International Federation of Gynecology and Obstetrics (FIGO) 2018 staging guidelines who underwent pelvic 3.0T magnetic resonance imaging (MRI), including MRI and DWI, were enrolled, and the diagnostic value of preoperative staging of cervical cancer was compared between the MRI and DWI groups. The DWI group was used to explore the potential association of mean $\mathrm{ADC}\left(\mathrm{ADC}_{\text {mean }}\right)$ with different pathological characteristics and receiver operating characteristic (ROC) curves of $\mathrm{ADC}_{\text {mean }}$ generated to predict the appropriate postoperative supplementary therapy.

Results: The diagnostic coincidence rate of DWI was higher than that of MRI in preoperative staging of cervical cancer $\left(\chi^{2}, \mathrm{P}<0.05\right)$ and determined as stages IB1 + IB2 + IIA1 (90.91\%), IB3 + IIA2 (93.48\%), and IIIC1p (95.16\%). The DWI staging results were consistent with postoperative pathological staging (Kappa value $=0.865, \mathrm{P}<0.001)$. We observed significant differences in $\mathrm{ADC}_{\text {mean }}$ values in relation to pathological type, histological grade, depth of stromal infiltration, tumor diameter, lymphovascular invasion, and pelvic lymph node metastasis of cervical cancer (all $\mathrm{P}<0.05$ ). The area under the ROC curve (AUC) was 0.815, with the best predictive value for postoperative supplementary therapy in cervical cancer (sensitivity $80.0 \%$, specificity $74.0 \%$ ) at $\mathrm{ADC}_{\text {mean }}$ of $0.910 \times 10^{-3} \mathrm{~mm}^{2} / \mathrm{s}$.

Conclusions: The DWI is a useful tool for preoperative evaluation of cervical cancer. In local cervical lesions, $\mathrm{ADC}_{\text {mean }}$ varies in relation to different clinicopathological characteristics and a reference index of $<0.910 \times 10^{-3} \mathrm{~mm}^{2} / \mathrm{s}$ can be effectively applied to predict the need for postoperative supplementary therapy.

Keywords: Diffusion-weighted imaging (DWI); cervical cancer; stage; apparent diffusion coefficient (ADC); supplementary therapy

Submitted Sep 06, 2021. Accepted for publication Nov 22, 2021.

doi: $10.21037 / \mathrm{atm}-21-5319$

View this article at: https://dx.doi.org/10.21037/atm-21-5319 


\section{Introduction}

Cervical cancer is one of the most common cancer types in women worldwide, ranking fourth in terms of both morbidity and mortality following breast, colorectal, and lung cancer, and remains the main cause of cancerassociated death in developing countries, posing a serious global threat to female health (1). In October 2018, the International Federation of Gynecology and Obstetrics (FIGO) terminated the era of continuous use of clinical staging and introduced postoperative pathological staging for cervical cancer. Since radiotherapy is one of the main treatment modalities for cervical cancer, imaging was proposed for use in staging for the first time, leading to substantial changes in diagnosis and treatment plans (2). The staging diagnosis of cervical cancer affects selection of clinical treatment options (surgical treatment, radiotherapy, or chemotherapy) while patients undergoing surgery require additional radiotherapy or chemotherapy if there are risk factors for postoperative pathology. For stage IB3/IIA2 cervical cancer, surgical treatment is currently considered to have similar efficacy as concurrent platinumbased chemoradiation (CCRT). The latest National Comprehensive Cancer Network (NCCN) guidelines still recommend CCRT as the first choice (category 1A option) for stage IB3/IIA2 as well as stage IIIC1 cervical cancer (3). For patients undergoing surgery, risk factors for supplementary therapy according to postoperative pathology need to be considered, which can inadvertently lead to superposition of invasive therapy. Effective preoperative prediction of the possibility of postoperative supplementary therapy may allow patients to select CCRT as the first treatment choice instead of surgery. Accurate preoperative assessment is therefore critical for appropriate selection of initial treatments for cervical cancer. In the past, clinical staging and evaluation of cervical cancer has been mainly based on gynecological examinations. With the continuous progress in medical imaging technology, evaluation of cervical cancer is increasingly dependent on imaging methods.

Magnetic resonance imaging (MRI) has unique advantages in soft tissue imaging (4) and is widely used in preoperative diagnosis of cervical cancer. Compared with other imaging methods, MRI has higher utility in pelvic evaluation and is effective in detection of primary tumor size, local diffusion, and lymph node metastasis, thus presenting a valuable means to monitor disease recurrence and treatment response. With recent advances in technology, MRI has gradually developed from morphological imaging to diffusion-weighted imaging (DWI) that can simultaneously perform functional imaging (5). Currently, DWI is the only noninvasive imaging procedure that can effectively detect the diffusion movement of water molecules in living bodies. The DWI method involves applying one gradient pulse pair of the same size, direction, and duration on both sides of the $180^{\circ}$ scan pulse and collecting echo signals to form images. Diffusion of water molecules results in weakening of tissue signal intensity. The degree of weakening is associated with the speed of molecular diffusion of water. Under conditions of limited diffusion movement of water molecules between tissues, the signal intensity is stronger. Changes in the signal intensity of tissues indirectly reflect the movement of water molecules within tissues under physiological and pathological conditions at the molecular level, thus signifying changes in the morphology, structure, and function of tissues (6). In addition, DWI facilitates quantification of changes in the degree of water molecule diffusion motion through evaluation of the apparent diffusion coefficient (ADC) value that reflects tissue lesions (7), which can obtain $\mathrm{ADC}_{\text {mean }}$ from a region of interest (ROI) along the inner margin of the tumor area was manually placed on the ADC map. However, at present, application of DWI in cervical cancer is at the exploratory stage and the current clinical guidelines still recommend pelvic MRI for preoperative cervical local lesions (3). The diagnostic value of enhanced MRI is greater, but associated with higher cost and risk of contrast agent allergy in clinical applications. Therefore, DWI may be a preferable alternative to enhanced MRI, which would resolve the issue of contrast agent allergy.

There is a potential application for DWI in evaluation of pathological type, histological grade, and lymph node metastasis and monitoring the efficacy of radiotherapy and chemotherapy in cervical cancer. However, the research is less in the preoperative prediction of postoperative supplementary therapy for patients with cervical cancer, which fully combine imaging with clinical practice. And few studies to date have comprehensively analyzed correlations between DWI and clinicopathological characteristics. In particular, utilization of DWI to predict risk factors after surgery and guide clinical decision-making may be of significant value in selection of accurate treatment options for patients with cervical cancer. The main objective of the current study was to compare the diagnostic efficacy of DWI in preoperative staging of cervical cancer to that of MRI, analyze the correlation between ADC 
value and clinicopathological characteristics of cervical cancer, and explore the ability of preoperative DWI to predict whether supplementary treatment may be needed after surgery. Improved preoperative diagnostic staging should aid in the provision of optimized and personalized treatments for patients with cervical cancer. We present the following article in accordance with the STARD reporting checklist (available at https://atm.amegroups.com/article/ view/10.21037/atm-21-5319/rc).

\section{Methods}

\section{Patients}

The prospective study was conducted in accordance with the Declaration of Helsinki (as revised in 2013). The study was approved by the Institutional Board of Cancer Hospital of China Medical University, Shenyang, China (No. 2013010) and informed consent was taken from all individual participants. The inclusion criteria were as follows: (I) surgical treatment, (II) new cases without preoperative chemoradiotherapy, (III) MRI sequence and DWI sequence scanning completed preoperatively, and (IV) postoperative pathological diagnosis. The exclusion criteria were as follows: (I) any MRI examination contraindication, (II) excessive MRI artifacts affecting diagnosis, (III) non-surgical treatment of patients, and (IV) imperfect clinical data. A total of 273 cervical cancer patients aged 27-70 years (average age of $49.63 \pm 9.32$ years) were prospectively recruited from June 2014 to June 2017. According to MRI scan sequence patterns, participants were divided into MRI and DWI groups.

All participants were diagnosed based on their postoperative pathological results. The FIGO 2018 staging of participants revealed 165 stage IB1 + IB2 + IIA1, 46 stage IB3 + IIA2, and 62 stage IIIC1p cases. The clinicopathological characteristics included pathological type, histological grade, depth of stromal infiltration, tumor diameter, vaginal fornix invasion, lymphovascular invasion, and pelvic lymph node metastasis. Overall, 126 participants needed postoperative supplementary therapy, which was not required by the remaining 147 participants.

\section{Imaging and data acquisition}

Pelvic MRI examinations were performed using a 3.0-T unit (Magnetom Trio; Siemens Medical Solutions, Erlangen, Germany) with an 8-channel phased-array body coil and respiratory gating technology. The scanning range was from the upper edge of the iliac crest to the lower edge of pubic symphysis. Patients were in a supine position, with the head elevated and breathing kept as steady as possible to reduce disturbances caused by breathing movements. Prior to MRI examination, the patient was instructed to drink water to keep the bladder moderately filled and rest for 15-30 min. Intrauterine Any intrauterine device (IUD) was removed the day before examination.

The MRI sequence was as follows: transverse plane T1WI, TR5 $50 \mathrm{~ms}$, TE13 ms, slice thickness of $4 \mathrm{~mm}$, spacing of $1 \mathrm{~mm}$, field of vision of $400 \mathrm{~mm} \times 400 \mathrm{~mm}$, matrix of $195 \times 320$, excitation number of 2 , FSE T2WI in transverse and sagittal planes, TR550 ms, TE3 ms, layer thickness of $4 \mathrm{~mm}$, spacing of $1 \mathrm{~mm}$, visual field of $400 \mathrm{~mm}$ $\times 400 \mathrm{~mm}$, matrix of $202 \times 384$, and excitation number of 2 .

The DWI sequence was as follows: single excitation of SE-EPI sequence on the transverse plane, TR3500 ms, TE93 ms, layer thickness $4 \mathrm{~mm}$, spacing $1 \mathrm{~mm}$, field of vision $400 \mathrm{~mm} \times 400 \mathrm{~mm}$, and matrix $320 \times 256$. Diffusionsensitive gradients in 3 directions were selected, and $b$ values were 0,800 , and $1,000 \mathrm{~s} / \mathrm{mm}^{2}$, respectively.

The MRI data were post-processed using GE OmniKinetics software (GE Healthcare, Chicago, IL, USA). The left and right diameter and anteroposterior diameter of the largest plane of the lesion were measured on MRI and DWI sequences, respectively, and upper and lower diameters of the largest plane of lesion in the sagittal plane were evaluated parallel to the uterine cavity. Each diameter was measured 3 times and the average value obtained. A ROI along the inner margin of the tumor area was manually placed on the ADC map. The ROI located in the lesion was as large as possible to avoid areas of uneven signal, such as necrosis, cystic degeneration, and bleeding and the surrounding area of the lesion. We measured 3 continuous layers of lesion in each patient to obtain the mean apparent diffusion coefficient $\left(\mathrm{ADC}_{\text {mean }}\right)$ values using GE OmniKinetics software.

\section{Statistical analysis}

The software SPSS 25.0 (IBM Corp., Armonk, NY, USA) was used for statistical analysis. Quantitative data of $\mathrm{ADC}_{\text {mean }}$ skewness distribution were expressed as median and quaternary range. Skewness distribution data were compared using Mann-Whitney U or Kruskal-Wallis $\mathrm{H}$ test, and comparison of group design data performed via $t$-test. Count data or graded data were analyzed via Pearson's $\chi^{2}$ test and Fisher's exact probability method. 
Table 1 Comparison of MRI and DWI for preoperative staging diagnosis of cervical cancer $(\mathrm{N}, \%)$

\begin{tabular}{lccccc}
\hline Stages & $N$ & MRI & DWI & $\chi^{2}$ & P value \\
\hline IB1 + IB2 + IIA1 & 165 & $124(75.15)$ & $150(90.91)$ & 14.539 & $<0.001$ \\
IB3 + IIA2 & 46 & $31(67.39)$ & $43(93.48)$ & 9.946 & 0.002 \\
IIIC1p & 62 & $44(70.97)$ & $59(95.16)$ & 12.899 & $<0.001$ \\
Total & 273 & $199(72.89)$ & $252(92.31)$ & 35.797 & $<0.001$ \\
Kappa & 273 & 0.865 & 0.536 & 257.963 & $<0.001$ \\
\hline
\end{tabular}

P, Pearson's $\chi^{2}$ test; Fisher's exact test. MRI, magnetic resonance imaging; DWI, diffusion weighted imaging.

Table $2 \mathrm{ADC}_{\text {mean }}$ values at different stages of cervical cancer (N, IQR)

\begin{tabular}{lccc}
\hline Stages & $\mathrm{N}$ & $\mathrm{ADC}_{\text {mean }}\left(\times 10^{-3} \mathrm{~mm}^{2} / \mathrm{s}\right)$ & $\mathrm{P}$ value \\
\hline IB1 + IB2 + IIA1 & 165 & $0.975(0.910-1.096)$ & $<0.001$ \\
IB3 + IIA2 & 46 & $0.905(0.847-0.990)$ & \\
IIIC1p & 62 & $0.835(0.815-0.880)$ & \\
\hline
\end{tabular}

$\mathrm{P}$, Mann-Whitney $\mathrm{U}$ test. $A D C_{\text {mean }}$, mean apparent diffusion coefficient; IQR, interquartile range.

Receiver operating characteristic (ROC) curves were generated. Sensitivity, specificity, positive predictive value, negative predictive value, positive likelihood ratio, negative likelihood ratio, and accuracy were calculated. The Kappa test was used for consistency (Kappa value $>0.75$ indicated good consistency, $0.4-0.75$ moderate consistency, $<0.4$ poor consistency). We regarded $\mathrm{P}$ values $<0.05$ as statistically significant.

\section{Results}

\section{Comparison of MRI and DWI as assessment tools for cervical cancer}

Among the various stages of cervical cancer, we observed significant differences in the preoperative diagnostic coincidence rates between MRI and DWI groups $(\mathrm{P}<0.05)$. The diagnostic coincidence rates of DWI for each stage were markedly higher than those of MRI, as shown in Table 1 . Consistency analysis of MRI, DWI, and postoperative pathological stage groups showed higher consistency of DWI with Kappa value of 0.865 and MRI with Kappa value of $0.536(\mathrm{P}<0.05)$.

The $\mathrm{ADC}_{\text {mean }}$ showed a decreasing trend with increased cervical cancer FIGO (2018) stage. The $\mathrm{ADC}_{\text {mean }}$ at the late stage (IIIC1p) was significantly lower than that at the early $(\mathrm{IB} 1+\mathrm{IB} 2+\mathrm{IIA} 1)(\mathrm{P}<0.001)$ and locally advanced (IB3 + IIA2) stages $(\mathrm{P}<0.001)$. The $\mathrm{ADC}_{\text {mean }}$ at the locally advanced stage (IB3 + IIA2) was significantly lower than that at the early stage (IB1 + IB2 + IIA1) $(\mathrm{P}<0.001)$, as shown in Table 2 .

\section{Correlation of DWI with clinicopathological parameters}

The differences in $\mathrm{ADC}_{\text {mean }}$ values according to pathological type, histological grade, depth of stromal infiltration, tumor diameter, lymphovascular invasion, and pelvic lymph node metastasis were statistically significant $(\mathrm{P}<0.05$, Table 3). The $\mathrm{ADC}_{\text {mean }}$ of squamous cell carcinoma (SCC) was significantly lower than that of non-squamous cell carcinoma $(\mathrm{P}<0.05)$ and that of high-grade cancer was significantly higher than that of medium-low grade cancer $(\mathrm{P}<0.001)$. The $\mathrm{ADC}_{\text {mean }}$ of the group stratified by $>1 / 2$ depth of stromal infiltration was significantly lower than that of $\leq 1 / 2$ depth of stromal infiltration $(\mathrm{P}<0.001)$. The $\mathrm{ADC}_{\text {mean }}$ of tumor sizes $\geq 4 \mathrm{~cm}$ was significantly lower than that of tumors $<4 \mathrm{~cm}(\mathrm{P}<0.05)$ and that of lymphovascular invasion was significantly lower than nonlymphovascular invasion $(\mathrm{P}<0.001)$. The $\mathrm{ADC}_{\text {mean }}$ of the group with positive pelvic lymph nodes was markedly lower than that of the negative pelvic lymph node group $(\mathrm{P}<0.001)$. No significant differences between $\mathrm{ADC}_{\text {mean }}$ values of groups with vaginal fornix involvement were observed.

\section{Effectiveness of DWI in assessment of cervical cancer}

The diagnostic efficiency of preoperative staging was compared between MRI and DWI groups. The results showed that for different stages of cervical cancer, DWI was consistently higher than MRI in terms of diagnostic sensitivity, specificity, positive predictive value, negative predictive value, positive likelihood ratio, negative likelihood ratio, and accuracy (Table 4).

\section{Value of DWI in prediction of postoperative supplementary therapy for cervical cancer}

According to treatment criteria, participants were divided into two groups: (I) those requiring postoperative supplementary therapy and (II) those with no requirement for postoperative supplementary therapy.

Criteria for postoperative supplementary therapy were as follows: at least 1 existing high-risk factor (positive lymph 
Table $3 \mathrm{ADC}_{\text {mean }}$ in relation to clinicopathological characteristics

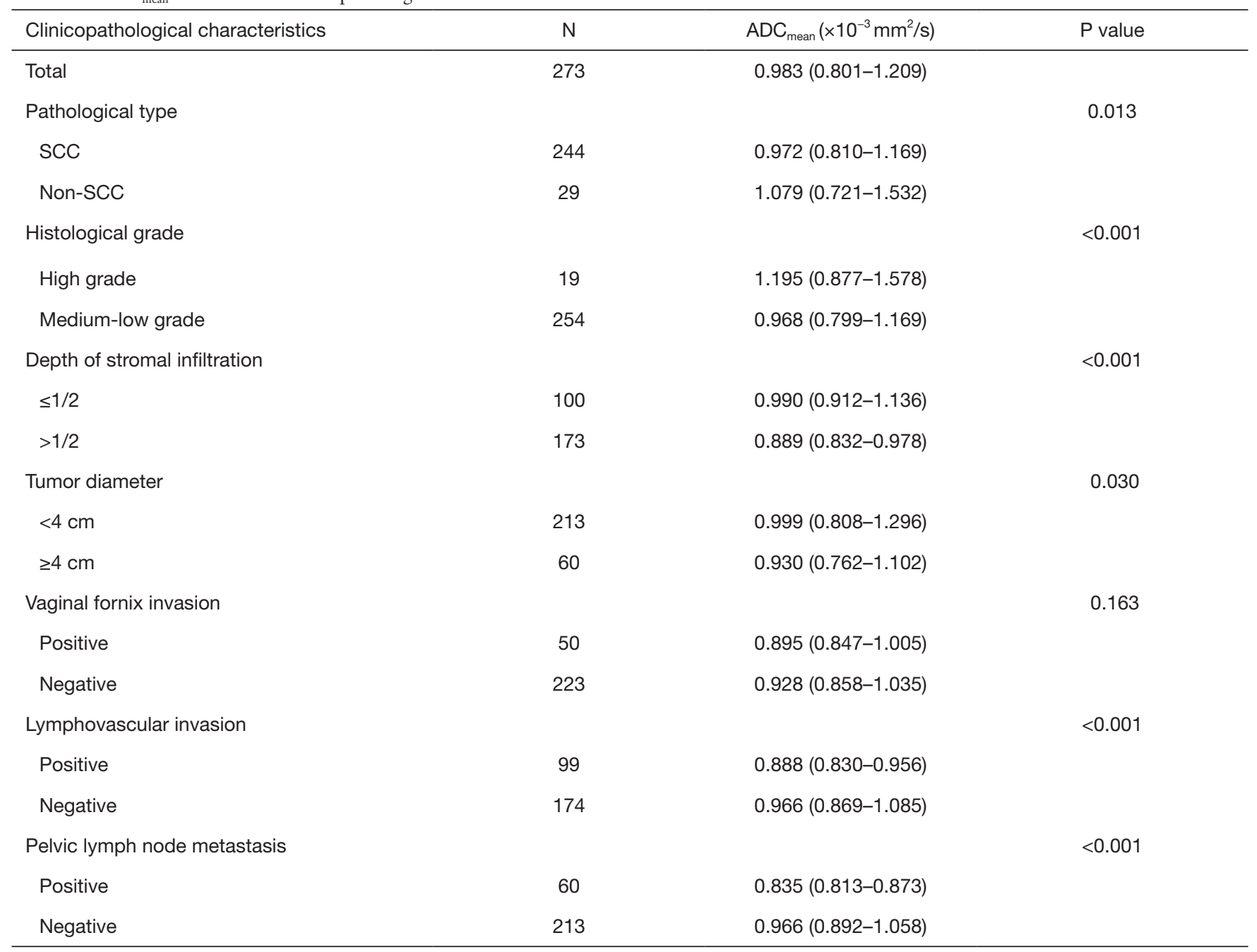

P, Mann-Whitney $U$ test. $A D C_{\text {mean }}$, mean apparent diffusion coefficient; SCC, squamous cell carcinoma.

Table 4 Comparison of MRI and DWI in preoperative staging diagnosis of cervical cancer

\begin{tabular}{|c|c|c|c|c|c|c|c|}
\hline Stages & SEN (\%) & SPE (\%) & $\mathrm{PV}^{+}(\%)$ & $\mathrm{PV}^{-}(\%)$ & $\mathrm{LR}^{+}$ & $\mathrm{LR}^{-}$ & Accuracy (\%) \\
\hline MRI & 75.15 & 79.63 & 84.93 & 67.72 & 3.69 & 0.31 & 76.92 \\
\hline DWI & 90.91 & 95.37 & 96.77 & 87.29 & 19.64 & 0.10 & 92.67 \\
\hline \multicolumn{8}{|c|}{ IB3 + IIA2 } \\
\hline DWI & 93.48 & 96.04 & 82.69 & 98.64 & 23.58 & 0.07 & 95.60 \\
\hline \multicolumn{8}{|l|}{ IIIC1p } \\
\hline MRI & 70.97 & 88.15 & 63.77 & 91.18 & 5.99 & 0.33 & 84.25 \\
\hline DWI & 95.16 & 96.68 & 89.39 & 98.55 & 28.68 & 0.05 & 96.34 \\
\hline
\end{tabular}

DWI, diffusion weighted imaging; MRI, magnetic resonance imaging; SEN, sensitivity; SPE, specificity; $\mathrm{PV}^{+}$, positive predictive value; $\mathrm{PV}^{-}$, negative predictive value; $\mathrm{LR}^{+}$, positive likelihood ratio; $\mathrm{LR}^{-}$, negative likelihood ratio. 
Table 5 Sedlis criteria for squamous cell carcinoma

\begin{tabular}{llc}
\hline $\begin{array}{l}\text { Lymphovascular } \\
\text { invasion }\end{array}$ & $\begin{array}{l}\text { Depth of stromal } \\
\text { infiltration }\end{array}$ & Tumor diameter $(\mathrm{cm})$ \\
\hline+ & Outer $1 / 3$ & Any size \\
+ & Medium $1 / 3$ & $\geq 2$ \\
+ & Inner $1 / 3$ & $\geq 5$ \\
- & Medium or outer $1 / 3$ & $\geq 4$ \\
\hline
\end{tabular}

+ , positive; -, negative.

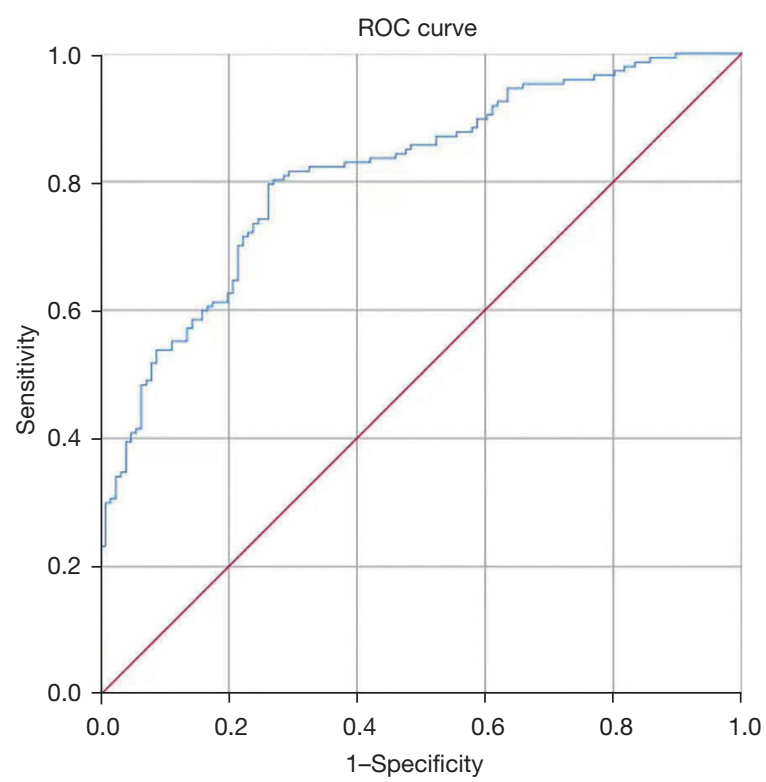

Figure 1 ROC curve of $\mathrm{ADC}_{\text {mean }}$ to predict the need for postoperative supplementary therapy. ROC, receiver operating characteristic; $\mathrm{ADC}_{\text {mean }}$, mean apparent diffusion coefficient.

node, positive incisor margin, and parauterine infiltration) or moderate risk factor according to Sedlis criteria in Table 5 (SCC).

According to the 4-factor model (non-squamous cell carcinoma), at least 2 factors should be present, specifically, adenocarcinoma or adenosquamous carcinoma, tumor diameter $>3 \mathrm{~cm}$, lymphovascular invasion, or tumor invasion of the outer $1 / 3$ depth of stromal infiltration.

Overall, 126 patients required postoperative supplementary therapy with $\mathrm{ADC}_{\text {mean }}$ of $0.871(0.825-$ $0.920) \times 10^{-3} \mathrm{~mm}^{2} / \mathrm{s}$ while 147 cases did not require postoperative supplementary therapy with $\mathrm{ADC}_{\text {mean }}$ of 0.996 $(0.916-1.120) \times 10^{-3} \mathrm{~mm}^{2} / \mathrm{s}, \mathrm{t}=-8.779(\mathrm{P}<0.001)$.

An $\mathrm{ROC}$ curve of $\mathrm{ADC}_{\text {mean }}$ was generated for predicting the requirement for postoperative supplementary therapy (Figure 1). The AUC area was 0.815 and the cut-off value of $\mathrm{ADC}_{\text {mean }}$ was $0.910 \times 10^{-3} \mathrm{~mm}^{2} / \mathrm{s}$, with a sensitivity of $80.0 \%$ and specificity of $74.0 \%$.

\section{Discussion}

The treatment methods of cervical cancer are relatively diverse, and surgery and radiotherapy currently have equivalent importance in the management of earlystage cancer. Minimization of the superposition of injury treatment is one of the key selection principles. Additionally, disease assessment is closely related to treatment options. Radical hysterectomy plus bilateral pelvic lymph node dissection is the preferred treatment modality for early cervical cancer patients (stages IB1, IB2, and IIA1) and has been shown to achieve satisfactory therapeutic effects. For IB3 stage cancer, the IIA2 period of initial treatment with radical surgery or chemoradiation used in patients with locally advanced cervical cancer is still controversial (8). Earlier results have suggested that the curative effect was similar but initial surgical treatment of cases with postoperative risk factors often necessitates supplementary therapy, and therefore, rather than improve survival rates of patients with locally advanced cervical cancer, the incidence of adverse reactions may increase. The NCCN guidelines of 2021 still recommend concurrent chemoradiotherapy (category 1A option) as the first treatment choice (3). In addition, the FIGO [2018] updated cervical cancer staging guidelines (2) include pelvic lymph node metastasis as stage IIIC1 and recommend concurrent radiotherapy and chemotherapy as the preferred treatment for patients with stage IIIC1 cervical cancer. To achieve optimal therapeutic effects and avoid side effects caused by dual treatment, accurate preoperative assessment is therefore crucial.

During preoperative assessment of cervical cancer, clinical examinations are considerably influenced by the clinical competence of doctors and other subjective factors, commonly resulting in failure to accurately assess tumor size and obtain information on tumor invasion into adjacent tissues (9). Imaging techniques counteract the deficiencies of clinical examinations by enabling visualization of local cervical lesions and pelvic conditions more objectively and the superior soft tissue resolution of MRI can provide enhanced details for cervical cancer staging diagnosis (10). The noninvasive functional MRI imaging technique, DWI, provides high quality visual images of tumors via high 
signal intensity and supplies quantitative information via measurement of ADC values, thus presenting a significant advantage in tumor detection (11).

The DWI and ADC also could be applied for breast cancer, neoplasm of nervous system, colorectal cancer, and endometrial carcinoma, the advantages were obvious in assessing local lesions and surrounding tissue, and has been evaluated for diagnosis of lymph node metastasis in these cancers. Preoperative imaging can be performed to evaluate tumor size, parauterine invasion, lymph node metastasis, and vaginal, bladder, and rectal invasion in cervical cancer. In MRI imaging, tumor invasion is usually defined by the presence or absence of rupture of the low-intensity cervical interstitial ring. However, due to the biological reaction of the surrounding cervical tissue caused by tumor growth, pressure, and edema, the low-strength ring is often destroyed by non-tumor tissue, leading to increased falsepositive results of MRI (12). In contrast, DWI reflects cell density based on the measurement of water diffusion movement. Compared with normal cervical stroma or parauterine tissue, active proliferation of malignant tumor cells leads to increased cell density, decreased extracellular space, increase in nucleo-cytoplasmic ratio, and increase in the limited degree of water molecule diffusion, presenting high signal intensity on the image. The employment of DWI facilitates accurate analysis of local lesions, surrounding tissue, and lymph node metastasis on images $(13,14)$. The quantitative index ADC value of DWI can be effectively applied to evaluate the degree of malignancy of cervical lesions (15). In a study by Demirbaş et al. (16) including 25 patients with cervical cancer, the ADC value was shown to provide valuable data for diagnosis and tumor staging of cervical cancer. In our prospective study, 273 patients with cervical cancer who underwent surgery were selected as the participants. Preoperative MRI and DWI examinations were performed and used for postoperative pathological diagnosis, which revealed significant advantages of DWI in preoperative staging diagnosis of cervical cancer. The benefits of DWI were compared in relation to MRI in preoperative staging diagnosis of early (IB1 + IB2 + IIA1), locally advanced (IB3 + IIA2), and advanced (IIIC1) cervical cancer. Overall, DWI staging showed better consistency with postoperative pathological staging results $(\mathrm{P}<0.001)$. The coincidence rates of DWI diagnosis staging were $90.91 \%, 93.48 \%$, and $95.16 \%$ while those of MRI diagnosis staging were $75.15 \%, 67.39 \%$ and $70.97 \%$, of early, locally advanced, and advanced cervical cancer, respectively $(\mathrm{P}<0.05)$. Simultaneously, $\mathrm{ADC}_{\text {mean }}$ values of local cervical lesions at different stages were significantly variable $(\mathrm{P}<0.001)$. Therefore, in addition to the advantages of imaging, the quantitative $\mathrm{ADC}_{\text {mean }}$ index of DWI provides an important reference value for preoperative staging diagnosis of cervical cancer (17). The overall sensitivity, specificity, positive predictive value, and negative predictive value of DWI in preoperative evaluation of cervical cancer were significantly greater than those of MRI. Therefore, DWI may serve as a critical guide for accurate preoperative staging and clinical decision-making.

The potential correlations between ADC value and various clinicopathological characteristics of cervical cancer have not been examined to date. In the numerical transformation of $\mathrm{ADC}, \mathrm{ADC}_{\text {mean }}$ reflects the whole tumor condition more completely (18). In this study, we systematically evaluated the association of $\mathrm{ADC}_{\text {mean }}$ with various pathological characteristics of cervical cancer. Notably, $\mathrm{ADC}_{\text {mean }}$ values for cervical cancer according to pathological type, histological grade, depth of stromal infiltration, tumor diameter, lymphovascular invasion, and pelvic lymph node metastasis were significantly different $(\mathrm{P}<0.05)$ and clinicopathological characteristics were closely associated with staging and prognosis, with important clinical implications. While earlier reports have supported the predictive value of $\mathrm{ADC}_{\text {mean }}$ of cervical cancer in pathological type and histological grade (19-21), consistent with our conclusions, this information was not considered the most valuable for preoperative cervical cancer patients with available tissue pathology. Our experiments showed that depth of stromal infiltration, tumor diameter, lymphovascular invasion, and pelvic lymph node metastasis evaluation with $\mathrm{DWI}$ is superior to $\mathrm{MRI}$, and $\mathrm{ADC}_{\text {mean }}$ values of these pathological factors provide important predictive parameters that can be effectively used to assess the need for postoperative supplementary radiotherapy or chemotherapy, which has important clinical implications. In particular, assessment of lesion sizes of endophytic cervical cancer is often erroneous, affecting the accurate selection of surgical methods, which remains an ongoing clinical challenge. Exner et al. (22) reported similar values of MRI and DWI in terms of tumor diameter evaluation. However, our results showed that $\mathrm{ADC}_{\text {mean }}$ of tumor diameters $\geq 4 \mathrm{~cm}$ was significantly lower than that of tumor diameters $<4 \mathrm{~cm}$ in the DWI group $(\mathrm{P}<0.05)$. Data from the current study support the utility of DWI and calculated $\mathrm{ADC}_{\text {mean }}$ as important guiding parameters for accurately assessing tumor load. In lymphatic or blood vessels, lymphovascular invasion is an important factor for distant metastasis of tumor cells 
and therefore incorporated in Sedlis evaluation criteria. Prediction of lymphovascular invasion preoperatively via noninvasive indexes is an important strategy to avoid injury due to excessive or inappropriate treatment. We recorded significantly lower $\mathrm{ADC}_{\text {mean }}$ of cases positive for lymphovascular invasion relative to those negative for lymphovascular invasion $(\mathrm{P}<0.001)$. Our data support the potential value of $\mathrm{ADC}_{\text {mean }}$ in preoperative prediction of lymphovascular invasion, consistent with earlier results (23). So far, pathological diagnosis is the only gold standard and the clinical utility of DWI in preoperative assessment of lymphovascular invasion requires further research attention. At the same time, DWI facilitates more accurate assessment of pelvic lymph node metastasis, further verifying the conclusions of relevant studies $(24,25)$, which support its importance in preoperative diagnosis of stage IIIC1r cervical cancer and selection of appropriate treatments.

A significant advantage in the treatment of cervical cancer is that in the main pathological type, SCC, radiotherapy has a definite effect and even more indications than surgery. Based on the basic principle of nonsuperimposed injury treatment, postoperative superimposed chemoradiotherapy should be avoided to reduce sideeffects in patients undergoing surgery. Halaska et al. (26) reported that complications of bladder, intestinal tract, and lower extremities after surgery for cervical cancer were significantly higher than those following radiation alone, and the incidence of edema of the lower extremities was as high as $47 \%$. In order to avoid injury due to treatment superposition, this study focused on preoperative assessment of cervical cancer and its utility in prediction of the necessity for postoperative supplementary therapy. According to criteria for postoperative supplementary therapy (3), participants were classified into groups requiring and not requiring supplementary therapy for analysis. Our data revealed significantly lower $\mathrm{ADC}_{\text {mean }}$ value of preoperative cervical lesions in the group requiring supplementary therapy $(\mathrm{P}<0.001)$. Simultaneously, the ROC curve of $\mathrm{ADC}_{\text {mean }}$ was generated to predict the requirement for postoperative supplementary therapy, which showed an AUC of 0.815. The predictive value was the highest when $\mathrm{ADC}_{\text {mean }}$ was $0.910 \times 10^{-3} \mathrm{~mm}^{2} / \mathrm{s}$, with a sensitivity of $80.0 \%$ and specificity of $74.0 \%$. Accordingly, we concluded that the risk of postoperative supplementary therapy was greater at $\mathrm{ADC}_{\text {mean }}$ values lower than $0.910 \times 10^{-3} \mathrm{~mm}^{2} / \mathrm{s}$ and recommended CCRT. Preoperative reference to $\mathrm{ADC}_{\text {mean }}$ of local cervical lesions should effectively allow individual patient selection of initial therapeutic treatment options that prevent potential injury caused by dual treatments. In this prospective study, we have proved the advantages of DWI and $\mathrm{ADC}$ images, however, and $\mathrm{ADC}_{\text {mean }}$ may be affected by different levels of diagnostic imaging physicians, we need to be validated in larger cohort. In conclusion, DWI was superior to MRI in preoperative evaluation of cervical cancer and the $\mathrm{ADC}_{\text {mean }}$ value of local cervical lesions differed according to various clinicopathological characteristics, thus providing an important reference for preoperative evaluation of cervical cancer. In particular, $\mathrm{ADC}_{\text {mean }}$ of local cervical lesions of $0.910 \times 10^{-3} \mathrm{~mm}^{2} / \mathrm{s}$ was identified as the highest value to predict requirement for postoperative supplementary therapy. Therefore, DWI may serve as an important tool for preoperative assessment to achieve optimized and personalized treatment of cervical cancer patients. We can evaluate the various clinicopathological characteristics in preoperative by DWI to achieve optimal therapeutic effects and avoid side effects caused by dual treatment for patients with cervical cancer.

\section{Acknowledgments}

The authors appreciate all the support provided by the Department of Gynecology, Radiology, and Nuclear Medicine, Cancer Hospital of China Medical University, Shenyang, China.

Funding: This work was supported by National Public Welfare Industry Research Fund (No. 201402020).

\section{Footnote}

Provenance and Peer Review: This article was commissioned by the editorial office, Annals of Translational Medicine for the series "New Progress and Challenge in Gynecological Cancer". The article has undergone external peer review.

Reporting Checklist: The authors have completed the STARD reporting checklist. Available at https://atm.amegroups. com/article/view/10.21037/atm-21-5319/rc

Data Sharing Statement: Available at https://atm.amegroups. com/article/view/10.21037/atm-21-5319/dss

Conflicts of Interest: All authors have completed the ICMJE uniform disclosure form (available at https://atm. amegroups.com/article/view/10.21037/atm-21-5319/coif). The series "New Progress and Challenge in Gynecological Cancer" was commissioned by the editorial office without 
any funding or sponsorship. DW served as the unpaid Guest Editor of the series. YL reports that this work was supported by National Public Welfare Industry Research Fund (No. 201402020 to YL). The authors have no other conflicts of interest to declare.

Ethical Statement: The authors are accountable for all aspects of the work in ensuring that questions related to the accuracy or integrity of any part of the work are appropriately investigated and resolved. The study was conducted in accordance with the Declaration of Helsinki (as revised in 2013). The study was approved by the Institutional Board of Cancer Hospital of China Medical University, Shenyang, China (No.: 2013010) and informed consent was taken from all individual participants.

Open Access Statement: This is an Open Access article distributed in accordance with the Creative Commons Attribution-NonCommercial-NoDerivs 4.0 International License (CC BY-NC-ND 4.0), which permits the noncommercial replication and distribution of the article with the strict proviso that no changes or edits are made and the original work is properly cited (including links to both the formal publication through the relevant DOI and the license). See: https://creativecommons.org/licenses/by-nc-nd/4.0/.

\section{References}

1. Bray F, Ferlay J, Soerjomataram I, et al. Global cancer statistics 2018: GLOBOCAN estimates of incidence and mortality worldwide for 36 cancers in 185 countries. CA Cancer J Clin 2018;68:394-424.

2. Bhatla N, Aoki D, Sharma DN, et al. Cancer of the cervix uteri. Int J Gynaecol Obstet 2018;143 Suppl 2:22-36.

3. Abu-Rustum NR, Yashar CM, Bean S, et al. NCCN Guidelines Insights: Cervical Cancer, Version 1.2020. J Natl Compr Canc Netw 2020;18:660-6.

4. Balcacer P, Shergill A, Litkouhi B. MRI of cervical cancer with a surgical perspective: staging, prognostic implications and pitfalls. Abdom Radiol (NY) 2019;44:2557-71.

5. Dappa E, Elger T, Hasenburg A, et al. The value of advanced MRI techniques in the assessment of cervical cancer: a review. Insights Imaging 2017;8:471-81.

6. Chen J, Zhang Y, Liang B, et al. The utility of diffusionweighted MR imaging in cervical cancer. Eur J Radiol 2010;74:e101-6.

7. Elsalam SMA, Mokhtar O, Adel L, et al. Impact of diffusion weighted magnetic resonance imaging in diagnosis of cervical cancer. Egyptian Journal of Radiology and Nuclear Medicine 2020. doi: 10.1186/s43055-0200144-2.

8. Gupta S, Maheshwari A, Parab P, et al. Neoadjuvant Chemotherapy Followed by Radical Surgery Versus Concomitant Chemotherapy and Radiotherapy in Patients With Stage IB2, IIA, or IIB Squamous Cervical Cancer: A Randomized Controlled Trial. J Clin Oncol 2018;36:1548-55.

9. Lindegaard JC, Petric P, Lindegaard AM, et al. Evaluation of a New Prognostic Tumor Score in Locally Advanced Cervical Cancer Integrating Clinical Examination and Magnetic Resonance Imaging. Int J Radiat Oncol Biol Phys 2020;106:754-63.

10. Wang X, Fan L, Yan W, et al. Comparison of accuracy and long-term prognosis between computed tomography-based and magnetic resonance imaging-based brachytherapy for cervical cancer: A meta-analysis. J Med Imaging Radiat Oncol 2020;64:151-62.

11. Traverso A, Kazmierski M, Welch ML, et al. Sensitivity of radiomic features to inter-observer variability and image pre-processing in Apparent Diffusion Coefficient (ADC) maps of cervix cancer patients. Radiother Oncol 2020;143:88-94.

12. Mongula JE, Bakers FCH, Mihl C, et al. Assessment of parametrial invasion of cervical carcinoma, the role of T2weighted MRI and diffusion weighted imaging with or without fusion. Clin Radiol 2019;74:790-6.

13. Lin $\mathrm{M}, \mathrm{Yu} \mathrm{X}$, Chen $\mathrm{Y}$, et al. Contribution of monoexponential, bi-exponential and stretched exponential model-based diffusion-weighted MR imaging in the diagnosis and differentiation of uterine cervical carcinoma. Eur Radiol 2017;27:2400-10.

14. Devine C, Gardner C, Sagebiel T, et al. Magnetic Resonance Imaging in the Diagnosis, Staging, and Surveillance of Cervical Carcinoma. Semin Ultrasound CT MR 2015;36:361-8.

15. Liu Y, Zhang Y, Cheng R, et al. Radiomics analysis of apparent diffusion coefficient in cervical cancer: A preliminary study on histological grade evaluation. J Magn Reson Imaging 2019;49:280-90.

16. Demirbaş T, Cimilli T, Bayramoğlu S, et al. Contribution of diffusion-weighted imaging to diagnosis and staging of cervical cancer. Balkan Med J 2014;31:154-7.

17. Gulati P, Agarwal A, Gulati V. Cervical Malignancies: Status of MRI. Indian J Gynecol Oncol 2020. doi: 10.1007/s40944-020-00437-0.

18. Liu Y, Ye Z, Sun H, et al. Clinical Application of Diffusion- 
Weighted Magnetic Resonance Imaging in Uterine Cervical Cancer. Int J Gynecol Cancer 2015;25:1073-8.

19. Kuang F, Ren J, Zhong Q, et al. The value of apparent diffusion coefficient in the assessment of cervical cancer. Eur Radiol 2013;23:1050-8.

20. Dong Y, Dong RT, Zhang XM, et al. Influence of menstrual status and pathological type on the apparent diffusion coefficient in cervical cancer: a primary study. Acta Radiol 2021;62:430-6.

21. Payne GS, Schmidt M, Morgan VA, et al. Evaluation of magnetic resonance diffusion and spectroscopy measurements as predictive biomarkers in stage 1 cervical cancer. Gynecol Oncol 2010;116:246-52.

22. Exner M, Kühn A, Stumpp P, et al. Value of diffusionweighted MRI in diagnosis of uterine cervical cancer: a prospective study evaluating the benefits of DWI compared to conventional MR sequences in a $3 \mathrm{~T}$ environment. Acta Radiol 2016;57:869-77.

Cite this article as: Liu L, Wang S, Yu T, Bai H, Liu J, Wang D, Luo Y. Value of diffusion-weighted imaging in preoperative evaluation and prediction of postoperative supplementary therapy for patients with cervical cancer. Ann Transl Med 2022;10(2):120. doi: 10.21037/atm-21-5319
23. Mi HL, Suo ST, Cheng JJ, et al. The invasion status of lymphovascular space and lymph nodes in cervical cancer assessed by mono-exponential and bi-exponential DWIrelated parameters. Clin Radiol 2020;75:763-71.

24. Xiao M, Yan B, Li Y, et al. Diagnostic performance of MR imaging in evaluating prognostic factors in patients with cervical cancer: a meta-analysis. Eur Radiol 2020;30:1405-18.

25. Lee J, Kim CK, Park SY. Histogram analysis of apparent diffusion coefficients for predicting pelvic lymph node metastasis in patients with uterine cervical cancer. MAGMA 2020;33:283-92.

26. Halaska MJ, Novackova M, Mala I, et al. A prospective study of postoperative lymphedema after surgery for cervical cancer. Int J Gynecol Cancer 2010;20:900-4.

(English Language Editor: J. Jones) 